\title{
Empirical formula for cross section of direct electron-impact ionization of ions
}

\author{
V A Bernshtam, Yu V Ralchenko and Y Maron \\ Physics Faculty, Weizmann Institute of Science, Rehovot 76100, Israel
}

Received 21 August 2000

\begin{abstract}
Plasma ionization composition and level population calculations require, in particular, the cross sections of direct ionization from each quantum state into each state which may be generated by means of removal of any electron. We analysed published data and propose here an empirical formula for cross sections of direct electron-impact ionization of positive atomic ions. The cross sections given by this formula are in satisfactory agreement with those calculated in the distorted-wave (DW) approximation; therefore, we believe that for any direct state-tostate ionization channel this formula provides a reasonably accurate prediction of the DW result. Comparisons with published data and with the Lotz formula are reported as well.
\end{abstract}

\section{Introduction}

A calculation of the distribution of atoms over their ionization stages and quantum states is one of the key steps in the modelling of astrophysical phenomena and laboratory plasma experiments. For brevity, the local distribution of atoms over their ionization stages and quantum states is called the plasma composition in the following. Except for plasmas close to the local thermodynamic equilibrium (LTE), the calculated composition depends on the collisional cross sections chosen.

In the present paper we discuss cross sections of direct (single-step) ionization of positive atomic ions with charge greater than 1 by electron impact. Any collision of this type may be shown by the scheme

$$
\mathrm{A}_{z, q}+\mathrm{e}^{-} \rightarrow \mathrm{A}_{z+1, q^{\prime}}+2 \mathrm{e}^{-}
$$

where the subscript $z=2,3,4, \ldots$ denotes an ionization stage of target-ion $\mathrm{A}_{z}$, the subscript $q$ denotes a state (energy level) to be ionized and the subscript $q^{\prime}$ denotes a state of product ion $\mathrm{A}_{z+1}$ just after removal of the electron.

The cross sections of direct transitions (1) are of primary importance for so-called collisional-radiative (CR) calculations which provide a composition history of non-LTE plasmas. With a sufficient number of quantum states included in the CR model, an accurate description of the composition history may be provided accounting for direct transitions only. Indirect channels $\dagger$ have to be considered as long as the intermediate states are missing in the CR model. Being included in the model, the intermediate state converts the indirect channel into a sequence of direct transitions.

$\dagger$ Such as, for example, (a) collisional excitation of an inner-shell electron, followed by autoionization of this state, or (b) collisional excitation, followed by collisional ionization of the excited state. 
For ions of any chemical element, the number of direct ionization transitions (1), provided with published quantal cross section calculations, is small in comparison with the total number of transitions required for $\mathrm{CR}$ calculations. Experimental measurements of ionization cross sections add very little to the knowledge of state-to-state transitions because each experiment yields a total cross section, which is a sum of contributions from all direct and indirect channels leading from $\mathrm{A}_{z, q}$ into all states of $\mathrm{A}_{z+1}$. The cross section of each direct transition (1) cannot be inferred from such experimental data, except for transitions from ground states of simple ions in the low-energy domain.

The shortage in the cross section data motivated a search for empirical formulae capable of reasonably accurate prediction for any transition. An overview of the empirical formulae available for electron-impact ionization is given in [1]. The most successful expression was proposed 30 years ago by Lotz [2,3] for total direct ionization cross sections $\sigma_{z q}^{i n z}(\varepsilon)$, i.e. for ionization into all possible final states $q^{\prime}$ of $\mathrm{A}_{z+1}$,

$$
\sigma_{z q}^{i n z}(\varepsilon)=4.5 \times 10^{-14} \sum_{k} \xi_{z q k} \frac{\ln \left(\varepsilon / I_{z q k}\right)}{\varepsilon I_{z q k}} \mathrm{~cm}^{2} .
$$

Here $\varepsilon$ is the incident electron energy, the subscript $k$ runs over all $n l$-subshells of $\mathrm{A}_{z, q}, I_{z q k}$ is the minimal energy required for ionization from state $q$ into state $k$ (which may be an excited state) and $\xi_{z q k}$ is the number of equivalent electrons in the $n l$-subshell which has to lose one electron for the transition (1). This relationship takes into account the Bethe asymptotic dependence for behaviour of the ionization cross sections for high incident electron energy $(\ln (\varepsilon) / \varepsilon)$ and used the following classical scaling rule:

$$
\sigma_{z q}^{i n z}(\varepsilon) I_{z q k}^{2}=f\left(\varepsilon / I_{z q k}\right) .
$$

For three decades the Lotz formula has been widely used for cross section estimates and plasma modelling. In the present paper we report on our attempt to find a more accurate empirical formula based on the more recent cross section data. We introduce two empirical parameters depending on the orbital angular momentum $l$ and determine recommended values for these parameters. The dependence of the direct ionization cross section on orbital angular momentum arises naturally in the basic theory of electron impact ionization (see, e.g., [4]).

\section{Direct-ionization cross sections}

We processed cross sections published in [5-29], selecting energy domains where the cross sections may be reliably associated with direct ionization from a certain $n l$-subshell. Most of the data were retrieved via the NIFS atomic database [30]. For the cross section of the direct transition (1) our analysis resulted in the expression

$$
\sigma_{z q q^{\prime}}^{i n z}(\varepsilon)=C_{l}\left(\frac{\mathrm{Ryd}}{I_{z q q^{\prime}}}\right)^{2-\delta_{l}} \xi_{z q q^{\prime}} b_{z q q^{\prime}} \frac{\ln \left(\varepsilon / I_{z q q^{\prime}}\right)}{\varepsilon / I_{z q q^{\prime}}}
$$

where Ryd $=13.6 \mathrm{eV}$ is the Rydberg constant, $b_{z q q^{\prime}}$ is the branching ratio $[4,31,32]$, i.e. the probability that the removal of an electron from a proper $n l$-subshell generates the $q^{\prime}$ state out of a few possible ones (say, removal of a $1 \mathrm{~s}$ electron from the $1 \mathrm{~s}^{2} 2 \mathrm{~s}$ configuration may result in $1 \mathrm{~s} 2 \mathrm{~s}{ }^{1} \mathrm{~S}$ and $1 \mathrm{~s} 2 \mathrm{~s}{ }^{3} \mathrm{~S}$ terms with branching ratios $\frac{1}{4}$ and $\frac{3}{4}$, respectively). Coefficients $C_{l}$ and $\delta_{l}$ are chosen from the best fit of expression (3) to direct ionization cross sections selected from [5-29] as explained above.

Analysis of these cross sections showed that the accuracy of expression (3) increases if coefficients $C_{l}$ and $\delta_{l}$ are allowed to depend on the orbital quantum number $l$ of the electron 
Table 1. Coefficients $C_{l}$ and $\delta_{l}$ required for practical use of formula (3).

\begin{tabular}{lll}
\hline$l$ & $C_{l}\left(\mathrm{~cm}^{2}\right)$ & $\delta_{l}$ \\
\hline $\mathrm{s}$ & $1.7794 \times 10^{-16}$ & 0.0471 \\
$\mathrm{p}$ & $2.1597 \times 10^{-16}$ & 0.0910 \\
$\mathrm{~d}$ & $1.2131 \times 10^{-16}$ & 0.3319 \\
\hline
\end{tabular}

to be removed. For $l=0,1,2$ the coefficients are given in table 1 . For ionization from states having $l>2$, published cross section data are much less abundant; therefore, coefficients $C_{l}$ and $\delta_{l}$ for $l>2$ are less reliable and are not included in the present paper.

\section{Accuracy of prediction and the importance for plasma composition modelling}

We mentioned that cross sections of direct state-to-state ionization (3) are required for plasma modelling, such as calculation of ionization composition, level populations, kinetic and transport coefficients, optical parameters, spectrograms, etc. On the other hand, for comparison with published cross section data we have to introduce a total direct ionization cross section

$$
\sigma_{z q}^{i n z}(\varepsilon)=\sum_{q^{\prime}} \sigma_{z q q^{\prime}}^{i n z}(\varepsilon)
$$

In this expression the sum is taken over all states (energy levels) reachable by direct removal of one electron from $\mathrm{A}_{z, q}(1)$.

To estimate the accuracy of formula (3) we compared cross sections given by formulae (3) and (4) with a few sequences of direct ionization cross sections, which were not used in the determination of the coefficients $C_{l}$ and $\delta_{l}$. For the first series of such comparisons we have chosen the distorted wave with exchange (DWE) and the configuration average distorted-wave (CADW) cross sections available in the Oak Ridge database [33]. Rich sequences of DW results are available for argon and iron, namely, total direct ionization cross sections of ions $\mathrm{Ar}^{2+}, \mathrm{Ar}^{3+}, \mathrm{Ar}^{4+}, \mathrm{Ar}^{5+}, \mathrm{Ar}^{6+}, \mathrm{Ar}^{7+}, \mathrm{Fe}^{2+}, \mathrm{Fe}^{5+}, \mathrm{Fe}^{6+}, \mathrm{Fe}^{9+}, \mathrm{Fe}^{11+}, \mathrm{Fe}^{13+}$ from their ground states $\dagger$. For each of the 12 ions, we compared (4) with DW for five energy points, chosen over all energy domains. The comparison showed that for 55 of the 60 points the difference is less than $20 \%$. The worst point shows $43 \%$. On a basis of this comparison we believe that the empirical formula (3) predicts DW results commonly to $\pm 20 \%$; although for, say, $10 \%$ of the cross sections the inaccuracy of the prediction may be larger in some energy domains. Results related to this comparison are presented in figure 1. In each plot, a label shows the target ion and references to DW data $[19,22,33,34]$ are presented by the full curve with open squares; the full curve shows a total direct ionization cross section calculated using equations (3) and (4).

Besides these comparisons with DW calculations, we estimated the accuracy of formula (4) relative to the Lotz formula. Comparison of both these formulae with total direct ionization cross sections selected from experimental and computational data [5-29] showed that on average (over all transitions and various energy ranges) formulae (3) and (4) are more accurate than formula (2). Namely, for ionization from s-subshells we found an $11 \%$ deviation versus $23 \%$, ionization from p-subshells showed $23 \%$ versus $24 \%$, and ionization from d-subshells showed $30 \%$ versus $47 \%$. We believe that higher, on average, accuracy is reached due to (a) the use of two parameters, $C_{l}$ and $\delta_{l}$, instead of one, (b) accounting for the $l$ dependence in these

$\uparrow$ The CADW cross section available for $\mathrm{Ar}^{8+}$ is not included in this list because it is modified to account for ionization from metastable levels as was necessary for comparison with certain experiments. The cross section for $\mathrm{Fe}^{11+}$ is included in the list, despite being used for the determination of the coefficients $C_{l}$ and $\delta_{l}$. 

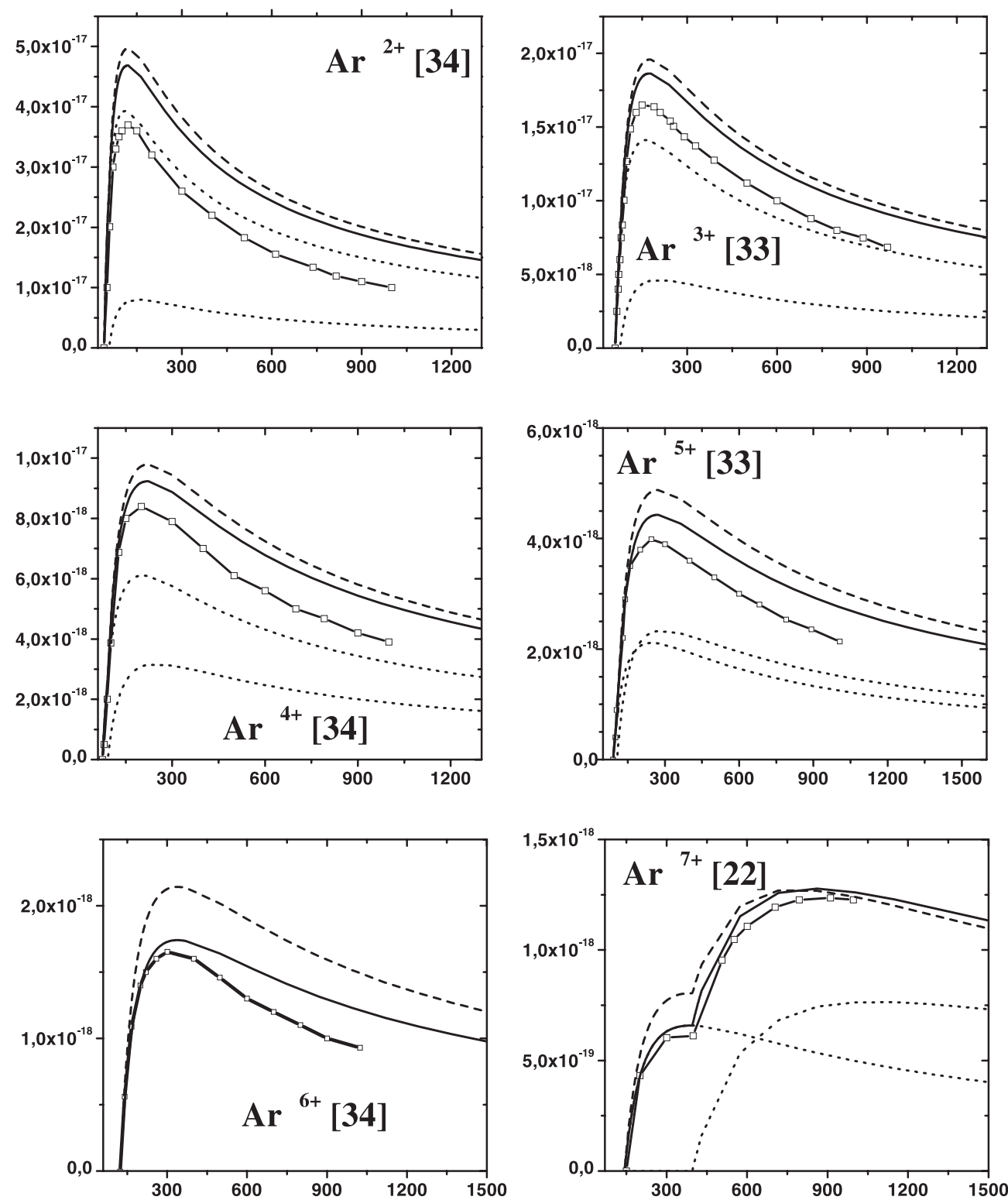

Figure 1. The total cross section corresponding to direct electron-impact ionization of $\mathrm{Ar}$ and $\mathrm{Fe}$ ions. The broken curves are results for Lotz' formula. The dotted curves are direct electron-impact ionization cross sections from individual subshells using formula (3), DW data are presented as full curves with open squares; the full curve shows the total direct ionization cross section calculated using equations (3) and (4).

parameters, (c) higher, on average, accuracy of modern cross section data in comparison with that available in the 1960s and (d) the increase in the total amount of published data [30,33].

The difference between the Lotz formula and the cross sections given by equation (3) becomes especially important for transient plasmas with rapidly varying parameters (e.g. fast $Z$-pinch plasmas [35]). As an example, we carried out a time-dependent collisional-radiative 

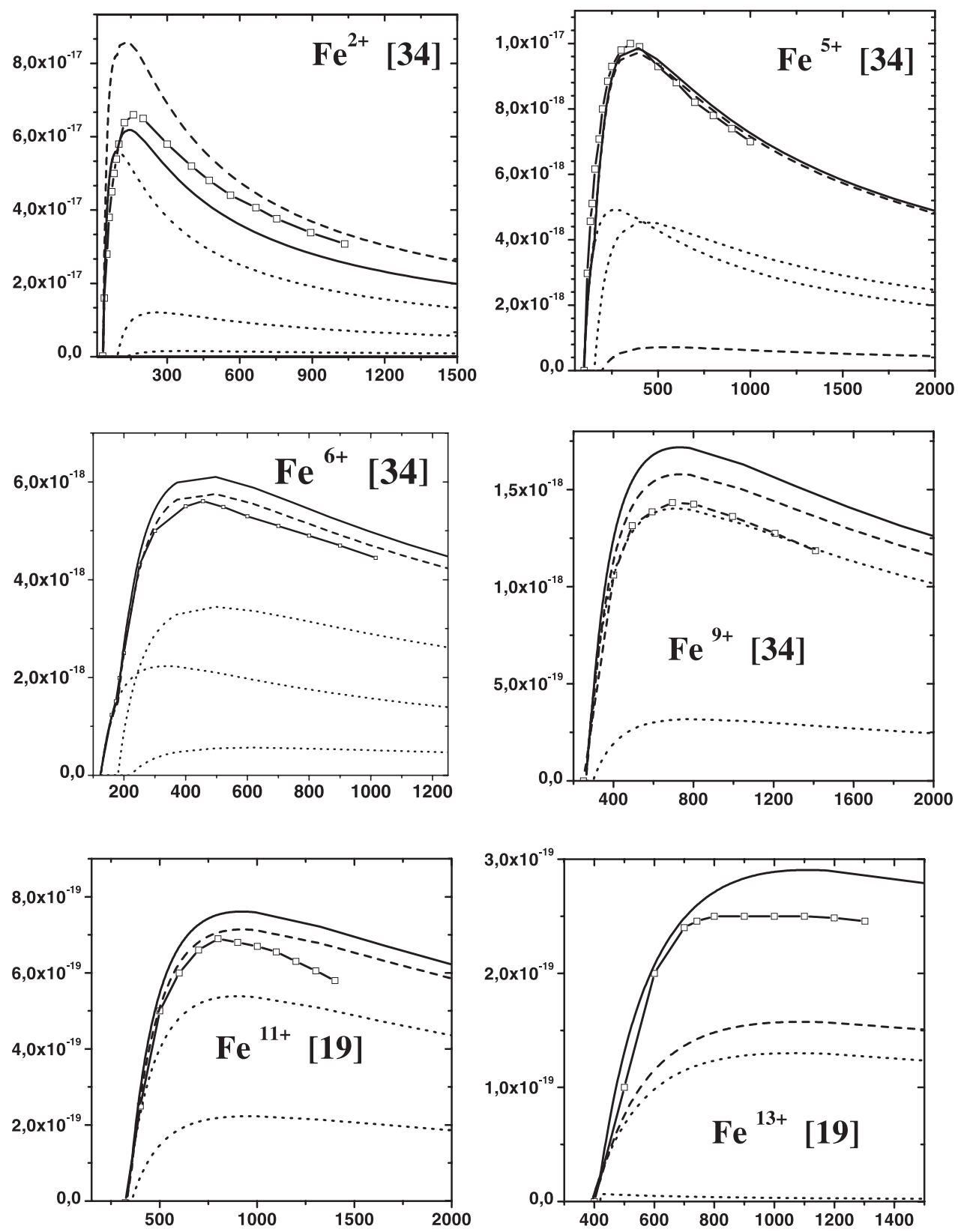

Figure 1. Continued.

simulation of a fast heated aluminium plasma with the constant ion density $n_{i}=10^{16} \mathrm{~cm}^{-3}$ and an electron temperature increasing linearly from 5 to $2500 \mathrm{eV}$ within $125 \mathrm{~ns}$. The electron density in this simulation was determined by ionization of the $\mathrm{Al}$ ions. It was found that the population of the term $2 \mathrm{~s} 2 \mathrm{p}^{2}{ }^{2} \mathrm{D}$ in $\mathrm{Al}$ Ix (the well known line with $\lambda=387.97 \AA$ originates from this term) differs by more than a factor of three depending on whether the Lotz formula or equation (3) is used in CR modelling (see figure 2(a)). In contrast, the population of the 

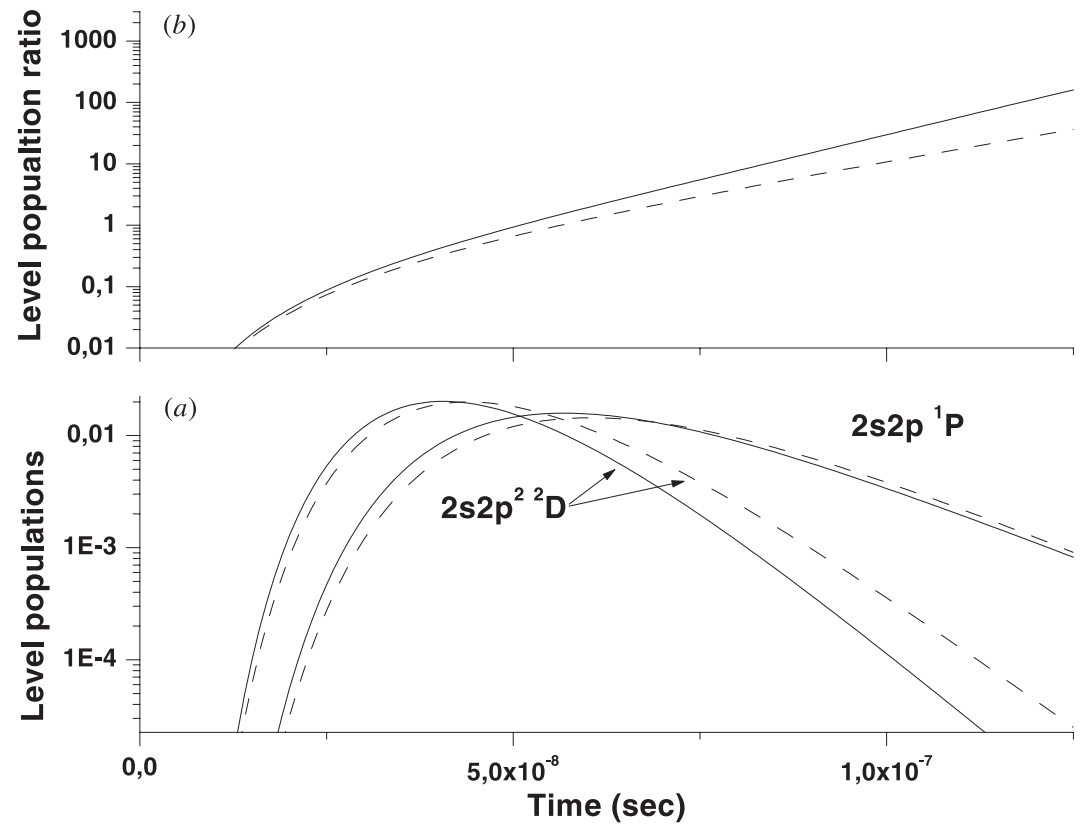

Figure 2. The results of level population calculations for different formulae for ionization cross sections for aluminium ions in a plasma with rapidly varying parameters. The broken curves are results for the Lotz formula.

term 2 s $2 \mathrm{p}{ }^{1} \mathrm{P}$ in $\mathrm{Al} x(\lambda=332.79 \AA)$ is almost insensitive to the choice of ionization cross sections. Thus, if the intensity ratio of this pair of spectral lines is used for determination of the electron temperature, as is often done in plasma spectroscopy [36], rather different results will be obtained for different choices of ionization cross sections (see figure $2(b)$ ). This conclusion may be valid for many other ratios of lines belonging to adjacent ions as well and therefore such a dependence of line ratios on ionization cross sections has to be taken into account in CR modelling.

\section{Summary}

Detailed CR calculations of ionization composition and level populations for non-LTE plasmas require the cross sections of direct state-to-state ionization (1). To match this need, we proposed here the empirical formula (3). Comparisons showed that cross sections given by this formula are close to those obtained in the DW approximation. On the basis of this comparison we believe that for ionization of any positive atomic ion with charge more than 1 from any state formula (3) provides a reasonably accurate prediction of the $D W$ result. The possibility of such a prediction is especially helpful for ionization from excited states and for ionization into excited states because for transitions of these types cross section data are practically missing.

Formulae (3) and (4) are applicable to any positive atomic ion $(z \geqslant 2)$, while the Lotz formula (2) is applicable to highly charged ions $(z \geqslant 4)$ only [2,3].

Formula (3) is designed as a convenient expression for the cross section of direct state-tostate ionization (1). By definition, this formula ignores contributions from indirect channels; therefore, formulae (3) and (4) underestimate total ionization cross sections of complex ions. A reader, who is interested in the reasonably accurate prediction of such cross sections, has to 
use the formula by Burgess and Chidichimo [37], or to perform elaborate quantal calculations. This comment relates to interpretation of experimentally determined cross sections as well.

Published cross section measurements are performed at low electron density $n_{\mathrm{e}}$ to exclude two-collision events. However, in plasmas of considerable electron density (say, at $n_{\mathrm{e}}>10^{19}$ e/cc) two-collisional removal of the outer-shell electron may have a higher probability than electron-impact excitation of the inner-shell electron, followed by autoionization. (Here, electron-impact excitation of the outer-shell electron, followed by removal of this electron in the other electron impact.) Moreover, the relative probability of autoionization decreases with $n_{\mathrm{e}}$ because of collisional de-excitation of the autoionizing level. (On the other hand, at sufficient electron density, collisional ionization of the autoionizing level also plays a role; thus, the kinetics becomes rather complicated and its consistent consideration requires CR calculations.) Thus, in a plasma the total ionization cross section may differ substantially from that found in the low-density limit. Therefore, prior to any application (even prior to an estimate of the ionization rate in a plasma of known composition) experimentally determined total ionization cross sections have to be analysed critically in respect of density effects. This remark does not relate to the use of experimental results in verifying quantal calculations because both correspond to the low-density limit of the total cross sections.

\section{Acknowledgments}

We are grateful to V I Fisher for valuable comments and suggestions. This work was supported by the Israel Academy of Science, Ministry of Absorption, and the Minerva Foundation, Munich, Germany.

\section{References}

[1] Younger S M and Märk T D 1985 Electron Impact Ionization ed T D Märk and G H Dunn (Vienna: Springer) ch $2, \mathrm{p} 24$

[2] Lotz W 1970 Z. Phys. 232101

Lotz W 1968 Z. Phys. 216241

Lotz W 1967 Z. Phys. 206205

[3] Müller A 1991 Physics of Ion Impact Phenomena ed D Mathur (Berlin: Springer) ch 2, p 13

[4] Sobelman I I, Vainshtein L A and Yukov E A 1981 Excitation of Atoms and Broadening of Spectral Lines (Berlin: Springer) (1995 2nd edn)

[5] Bray I Atomic Database (http://yin.ph.flinders.edu.au:8000/)

[6] Teng H 1999 J. Phys. B: At. Mol. Opt. Phys. 32443

[7] Chen C, Teng Z X, Yan S X, Wang Y S, Yang F J and Sun Y S 1998 At. Data Nucl. Data Tables 70255

[8] Chen C, Hu W, Fang D, Wang Y, Yang F and Teng H 1996 At. Data Nucl. Data Tables 64301

[9] Pindzola M S et al 1994 Phys. Rev. A 49933

[10] Gorczyca T W, Pindzola M S, Griffin D C and Badnell N R 1994 J. Phys. B: At. Mol. Opt. Phys. 272399

[11] Kao H C, Kuo T Y, Yen H P, Wei C M and Huang K N 1992 Phys. Rev. A 454646

[12] Pindzola M S 1991 Phys. Scr. 3735

[13] Pindzola M S, Griffin D C and Bottcher C 1990 Phys. Rev. A 411375

[14] Tinschert K, Muller A, Hofmann G, Huber K, Becker R, Gregory D C and Salzborn E 1989 J. Phys. B: At. Mol. Opt. Phys. 22531

[15] Shah M B, Elliott D S and Gilbody H B 1987 J. Phys. B: At. Mol. Phys. 203501

[16] Rinn K, Gregory D C, Wang L J, Phaneuf R A and Müller A 1987 Phys. Rev. A 36595

[17] Pindzola M S, Griffin D C, Bottcher C,Younger S M and Hunter H T 1987 Nucl. Fusion, Special Suppl. 21

[18] Crandall D H, Phaneuf R A, Gregory D C, Howald A M, Mueller D W, Morgan T J, Dunn G H, Griffin D C and Henry R J W 1986 Phys. Rev. A 341757

[19] Montague R G, Diserens M J and Harrison M F A 1984 J. Phys. B: At. Mol. Phys. 172085

[20] Younger S M 1983 J. Quant. Spectrosc. Radiat. Transfer 2961

[21] Younger S M 1982 Phys. Rev. A 253396 
[22] Younger S M 1981 Phys. Rev. A 241278

[23] Younger S M 1981 Phys. Rev. A 241272

[24] Younger S M 1981 Phys. Rev. A 231138

[25] Defrance P, Brouillard F, Claeys W and Van Wassenhove G 1981 J. Phys. B: At. Mol. Phys. 14103

[26] Younger S M 1980 Phys. Rev. A 22111

[27] Crandall D H, Phaneuf R A, Hasselquist B E and Gregory D C 1979 J. Phys. B: At. Mol. Phys. 12 L249

[28] Crandall D H et al 1978 Cross-section data in the Database of the Controlled Fusion Atomic Data Center of the Oak Ridge National Laboratory (http://www-cfadc.phy.ornl.gov/)

[29] McGuire E J 1977 Phys. Rev. A 1662

[30] Atomic Database of the National Institute of Fusion Science (NIFS), Nagoya, Japan (http://dbshino.nifs.ac.jp/) Also see Tawara H and Kato M 1998 Electron impact ionization data for atoms and ions Research Report (NIFS-data Series no 51)

[31] Sampson D H 1986 Phys. Rev. A 34986

[32] Foord M E and Nardi E 1990 J. Appl. Phys. 685028

[33] Atomic Database of the Controlled Fusion Atomic Data Center of the Oak Ridge National Laboratory (http://www-cfadc.phy.ornl.gov/)

[34] Younger S M 1981 At. Data Fusion 7190

[35] Reutov D D, Derzon M S and Matzen M K 2000 Rev. Mod. Phys. 72167

[36] Griem H R 1997 Principles of Plasma Spectroscopy (Cambridge: Cambridge University Press)

[37] Burgess A and Chidichimo M 1983 Mon. Not. R. Astron. Soc. 2031269 